\title{
"Hoy te siento... como algo más que una persona". Negociación de la paradoja de ser para uno mismo y para el otro ${ }^{1}$
}

\author{
Susanna Federici, Ph.D. ${ }^{2}$ \\ IARPP, ISIPSé, Roma
}

\begin{abstract}
Para Livia, el sentimiento de ser un "individuo" es ahora una especie de ancla que la retiene- a veces de forma dramática- para evitar que sea engullida por el torbellino de la fuerte dinámica de una familia fusional que está completamente dominada por la extrema fragilidad del hermano pequeño. En el trabajo analítico con éste último aparece que sentir como un individuo es un logro salvavidas difícil de volver a sentir cuando se exploran nuevas relaciones significantes que, de hecho, demuestran ser una fuente de gran estrés. Mediante esta presentación clínica se expone la forma en que experimentamos ser sujetos cuando tenemos la sensación de que somos alguien que puede distinguirse de los demás: este sentido de singularidad existe precisamente porque dialoga con la experiencia de pertenecer al colectivo.
\end{abstract}

\section{Palabras clave: Paradoja, Relacional, Pertenencia, Sí mismo}

For Livia the feeling of being an "individual" is actually a sort of anchor that she can hold on to sometimes dramatically - to keep from getting sucked into the maelstrom of the strong dynamics of a fusional family that is completely dominated by the extreme fragility of the younger brother. In the analytic work with him it emerges that feeling like an individual is a life-saving achievement that is hard to put back into play when exploring new significant relationships which, in fact, prove to be a source of great stress. Along this clinical presentation is discussed the way we experience being subjects when we have the feeling that we are someone who can be distinguished from others; this sense of uniqueness is such precisely because it dialogues with the experience of belonging to the collectivity.

Key Words: Paradox, Relational, Belonging, Self

English Title: "Today I feel you... as more of a person". Negotiation of the paradox of being for oneself and for the other.

\section{Cita bibliográfica / Reference citation:}

Federici, S. (2016). "Hoy te siento... como algo más que una persona". Negociación de la paradoja de ser para uno mismo y para el otro. Clínica e Investigación Relacional, 10 (1): 79-90. [ISSN 1988-2939] [Recuperado de www.ceir.info] DOI: 10.21110/19882939.2016.100105

\footnotetext{
${ }^{1}$ Presentado en la 6a Reunión anual de IARPP-España, Valencia. 24 de Octubre 2015. Traducción castellana de Alba Seoane.

${ }^{2}$ Miembro Didacta Fundador de ISIPSé (Istituto di Specializzazione in Psicologia del Sé e Psicoanalisi Relazionale, Roma, Italia). Ex-Presidenta de IARPP (International Association for Relational Psychoanalysis and Psychotherapy).
} 
Facilitar la llegada a la existencia de la otra persona, el sentimiento de que uno existe para uno mismo siendo consciente de la responsabilidad que tiene sobre el otro (Mitchell, 1997): un tema principal del psicoanálisis contemporáneo que a todos nos interesa. Mal Slavin (2007) ha explorado este tema desde una perspectiva evolutiva que pone en el centro de la capacidad de relacionarse la paradoja de ser para uno mismo y para el otro, como dijo "el escepticismo de un paciente es una capacidad adaptativa crucial". Sabiendo que compartiría esta conferencia con Mal, pensé que sería interesante discutir contigo un caso en el que esta paradoja está en el centro del proceso terapéutico.

Para Livia, el sentimiento de ser un "individuo" (la etimología de la palabra ser individuus, indivisible, "aquello que no divido con nadie más") es ahora una especie de ancla que la retienea veces de forma dramática- para evitar que sea engullida por el torbellino de la fuerte dinámica de una familia fusional que está completamente dominada por la extrema fragilidad del hermano pequeño. En el trabajo analítico con éste último aparece que sentir como un individuo es un logro salvavidas difícil de volver a sentir cuando se exploran nuevas relaciones significantes que, de hecho, demuestran ser una fuente de gran estrés.

En los primeros años de su análisis, nos enfocamos durante mucho tiempo en cómo podía estar ella más presente con su propia subjetividad, no en un aislamiento solitario sino en el aquí y ahora de las relaciones.

Después de un año y medio en terapia, un día le dije a Livia, con un sentimiento de alegría, "hoy te siento... cómo podría decirlo... como algo más que una "persona..."

Este comentario se escurrió en la sesión sin resonar de forma inmediata en nuestra conversación. Pero durante la siguiente sesión un área de investigación crucial se abrió ante nosotras como resultado de cómo fue poderoso y desestabilizador ese comentario para el paciente. En sus propias palabras, "cuando dijiste que "te siento como algo más que una persona" y después, recientemente, "hay una chica pequeña y muy insegura", Me sentí mal porque significaba que no me veías como una "persona" antes, y esto me hizo sentir avergonzada. Pero después me sentí mejor y también más fuerte."

Los desarrollos fueron sorprendentemente productivos y demostraron que los esfuerzos de la paciente para construirse a sí misma como individuo fueron recompensados por la ventaja de tener control, aunque a cambio de esconder un auténtico contacto con el otro y con la posibilidad de experimentar el hecho de ser ahora dos sujetos que se encuentran. Pronuncié esas palabras sin tener un intento terapéutico específico pero es cierto que el sentimiento de entrar finalmente en contacto con ella me hizo sentir mejor y compartí ese sentimiento con ella de pensar que era algo positivo para ambas: ipor fin entrábamos en contacto! Solo después- 
gracias a la paciente- me di cuenta de que expresando lo que habíamos conseguido de una forma positiva también estaba nombrando lo que había estado faltando durante un año y medio. Durante los meses previos yo había intentado probablemente establecer contacto y, aunque ella quería que llegaran a ella, ella había creído en el "personaje" que solía funcionarle mejor en sus contactos sociales. Ahora bien, podríamos describir la situación de otra forma a partir de esa observación, en el tratamiento psicoterapéutico, era posible encontrar no solo el campo intersubjetivo que incluía un estado del self que la hacía sentir con control y activa, pero también el campo intersubjetivo que incluía el estado del self de una niña de 3 años terriblemente asustada. En el pasado, solía definir el trabajo psicoterapéutico con el término "integración", que es el esfuerzo para acceder a un sentido del self más rico y cohesionado. Hoy encuentro más convincente el concreto de múltiples Sí mismos y la idea de que la terapia puede ayudar al paciente a que se mueva con más fluidez entre las diferentes configuraciones de los diferentes estados del self. Este enfoque es útil en la clínica porque ayuda al terapeuta a no quedarse estancado en una única manera de mirar al paciente, por ejemplo, como una "mujer joven que es eficiente y tiene el control" sino que lo fuerza a estar siempre abierto frente a la complejidad del sujeto.

Lo interesante es que mi postura no se guiaba según un plan prefijado que implicaba saber qué era lo mejor para el paciente. De algún modo, la base intersubjetiva que se desarrolló a lo largo del tiempo-y de la que la actitud terapéutica del analista es parte integral-permitió que emergieran momentos de encuentro, y que sucedieran algunos cambios.

Mientras con respecto a la contribución de mi subjetividad, el trabajo con Livia a veces me evocaba una configuración del sentido de mí misma que estaba íntimamente relacionado con mi historia relacional. Mi experiencia personal como una "niña sensible que no causaba problemas", a diferencia de mi hermano pequeño con sus ataques de cólera, a menudo resonaba. Sus berrinches mantenían a mi madre tan ocupada que sentía la necesidad de "no molestar" exponiéndome a mí misma continuamente al riesgo de no ser suficientemente vista. En estas situaciones caóticas y tensas también sentía la necesidad de separarme del contexto. Por eso también estaba tan interesada en encontrarme con la niña solitaria, asustada y enfadada en vez de tratar solo con la mujer joven, competente y sensible.

Aproximadamente cuatro años después de empezar el análisis Livia se mudó a un apartamento más grande y su novio de hecho se mudó con ella, aunque no de manera "oficial". El trabajo analítico intenso se empezó a enfocar entonces en su vida juntos. Livia piensa "mi casa es mía y él entra como un intruso". Discutimos durante mucho tiempo sobre la importancia de encontrar su propio espacio dentro de un espacio compartido. Aunque con altibajos, seguimos. 
Después de algunos meses, a la hora de nuestra sesión, recibo un mensaje suyo en mi móvil diciéndome que está a punto de dejar la casa pero no puede, se siente débil, "pero no es por ti", escribe. Después de pensar si sería mejor insistir, le mando un mensaje tranquilizador aplazando la cita para la próxima semana. En la próxima sesión, mientras espero que lleguellega tarde-me pregunto cómo está de enfadada conmigo en particular, teniendo en cuenta que pronto me iré fuera de la ciudad. Me remito a nuestro pasado reciente y me doy cuenta de que se esforzó por venir a nuestras últimas sesiones, diciendo que lo hacía por continuar más que porque en realidad le apeteciera. Entonces, al final de estas sesiones ella siempre dijo, "me alegro de haber venido, fue muy útil".

Pero quizá algo se está moviendo de forma inconsciente, algo que es mucho más fuerte y complejo de lo que pensamos o podemos pensar ahora. El análisis la lleva a dejar de tener sus usuales sueños de grandeza, como "seré la mejor y ganaré un premio por mi trabajo" o "encontraré el hombre perfecto que se entregará por completo a mí" etc. La niña que está aterrorizada y triste o enfadada y dominante está en cambio oponiendo una resistencia feroz, no quiere bajar a la tierra porque siente que eso significaría ser "sensible" de nuevo. Mientras espero, tomo notas, pensando en ella. Unos veinte minutos después decido llamarla. Al teléfono, Livia parece realmente sorprendida y desprevenida porque está convencida de que esta es la semana en la que yo estaría fuera de la ciudad. Bromeo y le digo, "¡debes sentirte realmente aliviada pensando que me marcho!"

Consideramos si deberíamos seguir hablando por teléfono o aplazar la sesión. Al final, decidimos que debería venir a las 5 pm ese mismo día, también porque le digo que preferiría verla en persona. Pienso en lo intenso que ha sido el análisis últimamente.

A las 5 p.m. se sienta en el sofá y después de algunos formalismos se pone a llorar casi de inmediato diciendo que siente que no tiene nada, que está totalmente expuesta; y lo más importante, dice: "últimamente no me encuentro a mí misma, estoy en un vacío."

Hablamos sobre ese vacío; no es tanto un sentido de soledad provocado por los muchos cambios sucedidos en su vida. Ella misma admite que es más que un vacío profundo y existencial; no puede encontrar un sentido subjetivo de sí misma que la guíe en sus decisiones.

Sobre todo, ahora que podría empezar partiendo de sus propios deseos y sueños, no puede encontrarse a sí misma, no sabe cómo y esto le produce una gran ansiedad, se siente tremendamente frágil. Esto la hace sentirse enferma físicamente, todo le empieza a producir alergia. En vez de ayudar, el análisis parece empeorar las cosas. Series de pensamientos, paralelos e interconectados salen entrecruzados. Poco a poco, empezamos a resolver las cosas. 
"No sé cómo abordar ese sentido de "madre" que necesito tanto, no sé cómo hacerlo. Aplazo mi sesión de psicoanálisis para cuando el trabajo sea "más fácil" porque temo que no podremos trabajar de forma eficiente después de esto. "

Imaginamos juntas que la sesión "imposible" para ella fue la que volvimos a aplazar otro día diferente del usual, en realidad, para ajustarse a una petición suya que sin embargo la expuso al riesgo de "sentirse demasiado desestabilizada".

Parece ser que Livia ha conseguido garantizarse a sí misma varias fuentes de validación afectiva (sus compañeros de trabajo, su novio, su analista) pero nunca se ha dejado llevar por completo, rendirse completamente a sí misma.

Menciono la transferencia sugiriendo que-a pesar de lo que sucedió con su madre-le doy libertad, en realidad velo por sus necesidades y deseos, no le digo lo que debería hacer, poniendo mi bienestar personal en primer plano. Exploramos sus sentimientos sobre mí y dice, "perdóname por decir esto porque es importante para mí pero a veces no encuentro ayuda tampoco aqui". Me pregunta si me siento enfadada o herida o desestabilizada por su comportamiento. Le contesto, "no tienes que protegerme". Como comprobaremos más adelante, Livia observa que ahora se da cuenta de cuánto está totalmente enfocada en el otro. Admite que esto lo ha experimentado casi con todo el mundo. Cuando está con alguien que significa algo para ella, ella no existe-el vacío-está totalmente enfocada en el otro. Por eso, al principio las relaciones sociales parecen ir bien y después aparecen los problemas. En lugar de experimentar realmente las cosas, tiene que estar sola, encerrada en su "pequeña habitación", a veces, en soledad, vuelve a rememorar lo que acaba de suceder y solo entonces "siente" lo que acaba de experimentar con alguien más. Es como si no estuviera dentro de su cuerpo y estuviera en otro lugar. La situación, de la que parece emerger solo de forma esporádica, le causa mucho dolor.

Cuando hablamos de que ella no ha recibido "alimento" de nuestro vínculo- o al menos no el necesario- no me siento preocupada o ansiosa (como lo habría estado en el pasado); hoy tengo el valor de afrontar las cosas como son y no me siento herida o abandonada, débil. Estoy realmente aquí para ella hoy. Le digo "lo que podemos decir en cambio es que la familiaridad ha crecido con los años y esto es algo importante que existe entre nosotras".

A veces, Livia me dice "por favor, dímelo" "dime lo que realmente siento porque no lo sé". Ahora ella cree en mí y en nuestro trabajo, guiada por la necesidad de encontrar una forma y una definición a sí misma. Me responsabilizo de no esquivar la cuestión y no contesto con preguntas de "analista neutral", pero tengo también la impresión de que podríamos correr el riesgo de repetir el tema transferencial de encontrar motivación solo en "agradarme". 
Livia recuerda la película "la Leyenda de 1900" (Tornatore, 1998) porque el final describe perfectamente cómo se siente. El pianista-que nació y pasó toda su vida en un transatlántico sin salir porque no tenía papeles-explica por qué no podía bajarse del barco en Nueva York, aunque finalmente consigue hacerlo después de muchos años.

Dice, "he aprendido a vivir entre la proa y la poa, he hecho que la gente baile, participe [...] Pero la tierra es un barco demasiado grande para mí porque hay "infinitas posibilidades".

Livia advierte que tiene la capacidad de encontrar el infinito (soñar, planear, sentirse viva) solo si hay límites claros, que en realidad corresponde a lo que el otro le proporciona. Recordamos las dificultades que experimentó en el pasado: ser sensata, no llorar para no preocupar a papá, portarse bien porque mamá tiene que cuidar a su hermano pequeño, conformándose con el tiempo que tenía disponible. Si no hay límites, pierde sus modales y se vuelve desorganizada.

Añado que tal vez el análisis resulte estresante precisamente porque no es concluyente.

No le digo qué hacer o qué decisiones tomar en su vida; quiero aceptar cómo se siente ella aquí y ahora, incluso con ese sentimiento tan pesado de "vacío". Ambas hemos trabajado duro y quizá tenemos que dejar que el péndulo del proceso se balancee un poco entre crear un tejido de conexión entre nosotras y la búsqueda de existencia para el alma, el origen del universo. Ya hemos experimentado estas oscilaciones, que han sido expresadas a menudo por su postura en el sofá en el que se va sentando y tumbando.

Livia confirma que hay algo que está entre estar completamente centrada en el otro y estar aislada, sola. Veremos a dónde nos lleva este camino intermedio. Al final de la sesión Livia me dice "gracias por llamarme".

Durante una sesión más reciente, Livia se tumba y dice: "hoy deberíamos hablar de algo totalmente diferente pero estoy en el fondo del pozo y no puedo salir. Me he estado sintiendo de este modo desde ayer y no puedo salir de esto. Lo intento, pero el sentimiento vuelve. Estoy como en un estado de completa depresión donde nada puede moverse. Todo va a cámara lenta."

Le ha costado encontrar las palabras, ha hecho un tremendo esfuerzo para describir el estado en el que todavía en parte se encuentra y quiere traerlo a nuestra sesión porque sabe que puede ayudarle, pero es muy duro para ella estar en contacto con eso. Un dolor enorme, profundo. Un sentido de muerte de "tumba". Las palabras "muerte" y "tumba" son las palabras que más se le ocurren. Llora, se disculpa, todavía necesita derramar muchas lágrimas:

"No he llorado todas las lágrimas de ese pozo." 
Intento estar ahí, cerca de ella, más callada que de costumbre, concentrada con mi cuerpo. Intento no seguir mi mente, que busca enseguida palabras y explicaciones, consuelos; intento estar ahí en ese momento, lo suficientemente firme. No me asusta cometer un error. El único problema para mí es que me gustaría sentir su dolor de una forma más personal, o debería decir acceder con más facilidad a la experiencia personal que es parecido a la que está describiendo. Pero ahora esto es lo que tengo.

Me pregunto si será como si las manos que se acercan al pozo para sacarla del pozo, las manos de hoy-las mías y las de su novio- están aún demasiado altas y ella no puede alcanzarlas y agarrarse a ellas.

Livia: "la cuestión es, no he llegado al fondo del pozo todavía. Es como si existiera el riesgo de no volver atrás". Hablamos de una experiencia de "aniquilación", ¿esto es quizá lo que ella quería decir cuando usaba la palabra "tumba"?

Mis oídos están zumbando como si en la habitación empezara a dilatarse y estuviera completamente aislada del resto del mundo. Un silencio ensordecedor, me digo a mí misma: "no te preocupes, no hay prisa".

El episodio que estresa a Livia es una cita que su hermano y su madre supuestamente tienen con un doctor recomendado por Livia. Su madre y su hermana han intentado ir ya tres veces sin éxito, de modo que pidieron a Livia que las llevara. Aunque a Livia no le gusta la idea de que la arrastren de ese modo, ha pedido mucho tiempo libre en su trabajo para poder hacerlo. Su madre la llama para decirle que ha pensado sobre eso toda la noche y se da cuenta de que necesita más tiempo para que Livia pueda llegar diez minutos antes de lo acordado (esto complica aún más las cosas a Livia). El tono de la llamada es el de cuando su madre está en una especie de pánico obsesivo. Hemos hablado a menudo de las maneras despóticas, frágiles y obsesivas de su padre, pero ahora nos damos cuenta de que, a su modo, su madre es igual, y ha empeorado mucho últimamente desde que su hermana se ha vuelto cada vez más extenuante.

Livia quería rechazar la petición de su madre, pero, poniéndose a sí misma casi en la posición de una salvadora que debe contener un ataque de pánico, acepta.

En otro nivel-que surgió con mucha fuerza esta vez-Livia se sintió completamente anulada.

Livia: "no sería suficiente decir que me siento "no vista", sería más bien "asesinada", "tengo que morir" como si fuera solo una cosa, un instrumento que se usara para tratar el pánico, y no puedo salir de eso porque el mensaje transmitido es: "si te vas, me moriré."

SF: "quizá has vivido siempre así con tus padres." 
Livia: "mamá siempre ha sido así. Podría haber sucedido en cualquier momento. Me encerraría en mi habitación para hacer mis deberes esperando que no viniera a llamar a la puerta. Porque podría tener que confrontarme con este terror o podría simplemente traerme un vaso de zumo y preguntarme sobre mis deberes. Incluso después, cuando volvía a casa de la universidad, no sabía lo que me encontraría. El hecho de que estuviera enfrentándome a mis exámenes finales parecía no importar."

SF: "como si eso fuera la alternativa", "existo a costa de tu propia muerte."

Livia: "quizá incluso antes porque ya estás muerta. Solo puedes ser un robot que hace lo que mamá dice, y, solo quizá, las cosas mejorarán de nuevo."

Seguimos reflexionando: quizá disociaron con el pánico y en ese momento la propia Livia entraría en un estado de profunda disociación. Durante este momento de la sesión Livia se ha tumbado en el sofá pero entonces se levanta.

Livia, adoptando una postura más firme y con un sentimiento de autocontrol, concluye: "mi única salvación es mi independencia financiera porque si dependiera lo más mínimo de ellos acabaría de nuevo absorbida por ese remolino."

De modo que volvimos al tema que surgió durante el primer año de análisis: para enfrentarse al terror de salir al mundo sintiéndose completamente desprovista de un sentido nuclear de sí misma, Livia ha creado una persona/máscara que podría funcionar, aprendiendo a interpretar bien el papel de la niña estudiosa y más tarde de la profesional competente. El precio a nivel afectivo fue considerable, solo con análisis fue posible empezar a contactar con estados del self disociados y no formulados de nuevo.

Poco a poco, conectamos con el sentido de tener que "aniquilarse" a sí misma para el beneficio del otro con el lugar especial que ella tenía con su padre, "la pequeña novia de papá". De hecho, con demasiada frecuencia, su función "especial" consistía en escuchar las historias de su padre, que la había elegido para ser testigo de su propia infancia dramática. Impuso sus historias terroríficas sobre las terribles injusticias que sufrió. La madre de su padre murió dándole a luz de modo que creció en un orfanato: cuando era pequeño lo dejaron en un centro para niños donde pasaban cosas terribles. Incluso cuando la guerra acabó las cosas no mejoraron porque su padre había formado una nueva familia a la que él no pertenecía. Solo cuando consiguió un buen trabajo a una edad muy temprana pudo finalmente experimentar una cierta seguridad.

Cuando Livia era una niña, escuchó todas estas historias y probablemente sintió todo el terror de su padre sin ningún filtro pero también se sentía orgullosa por haber sido escogida como testigo y como la persona que podría salvarle o al menos consolarle. A lo largo de nuestro largo trabajo analítico ella pudo volver a los tiempos en que tenía que "entregarse" a sí misma a ese 
tipo especial de escucha, cuando papá estaba inmerso en estas historias como si estuviera reviviéndolas y no pudiera ser interrumpido porque sería muy malo sacarle de ahí a la fuerza; Livia se volvió muy buena captando sus diferentes estados de ánimo.

Parece como si fuera la descripción de un campo intersubjetivo en el que los estados disociados del padre eran revividos a través de ser compartidos con su hija, probablemente yendo de la permeabilidad a la disociación por parte de la niña. Transmití a Livia la idea de que arrastraba los traumas de su padre en lo que hoy llamamos transmisión intergeneracional del trauma. Esto la ayudó a comprender ciertas situaciones en las que se encontró a sí misma experimentando estados inexpresables de desesperación y dolor como si estuviera viviendo de forma generalizada una angustia que no le pertenecía.

Daniel Stern (2004) se centró en el estudio de compartir los estados afectivos, que considera la forma más importante de participación de las experiencias subjetivas. Su conceptualización del proceso de intercambio intersubjetivo como "ajuste afectivo" se basa en la identificación de correspondencias de modalidad entrecruzada de las características temporales, en forma de intensidad de comportamientos del niño y el adulto. Stern también destaca El "peso" del cuidador en la experiencia intersubjetiva del niño a través de la consideración de que las motivaciones e intenciones socio-afectivas, como constantes elementos del medio social del niño, pronto se convertirán en la base de las expectativas que el pequeño desarrollará en sus relaciones con los demás.

La capacidad de leer las intenciones aparece muy pronto en el niño. En 2004 Stern escribió: "vemos el mundo humano según las intenciones y actuamos según nuestras propias intenciones. No puedes funcionar con otros humanos sin leer o deducir sus motivos o intenciones. Esta lectura o atribución de intenciones es nuestra guía más básica para responder e iniciar una acción. La deducción de las intenciones del comportamiento humano parece ser algo universal.

"Es un estado mental primitivo" (D. Stern, 2004, p.87).

Los enfoques de Trevarthen, Fogel, Tronick, Beebe se centran en las dinámicas de la comunicación como "coordinación intersubjetiva" (Trevarthen, 1998), "co-regulación" (Fogel, 1992) o "regulación mutua" (Tronick 2008, and Beebe et al., 2008). La investigación de Fogel y Beebe muestra que el sentido de sí mismo como agente está profundamente enraizado en la co-regulación de la "regulación interactiva" experimentada con el adulto significante, aclarando cómo es necesario para el niño desarrollar un sentido de continuidad de su intersubjetividad experimentada como parte integral de su no obstante primitivo sentido de sí mismo. 
Experimentamos ser sujetos cuando tenemos la sensación de que somos alguien que puede distinguirse de los demás: este sentido de singularidad existe precisamente porque dialoga con la experiencia de pertenecer al colectivo.

El sufrimiento puede aparecer cuando un paciente, en vez de vivir una dialéctica fluida entre la singularidad y la pertenencia, es forzado por su historia relacional a consolidar una organización más rígida y obligatoria. Un contexto patológicamente falto de límites entre los miembros de la familia ha llevado al paciente a buscar alivio encerrándose a sí misma en un horizonte autoreferencial. En realidad, Livia sufrió por la imposibilidad de experimentar de forma fluida y sin ansiedad la dialéctica entre singularidad y pertenencia. Básicamente, yendo a análisis quería cambiar algo, pero cómo. Si el cuidado está en la relación ¿cómo podemos facilitar el proceso de co-crear "formas de estar con" que permitan al paciente tener experiencias relacionales que no sean fuente de una ansiedad demasiado intensa? ¿Cómo podemos conseguirlo de una manera específica?

Un enfoque clínico basado en el reconocimiento de múltiples estados del self (Bromberg, 1998), y en la atención a una comunicación implícita y procesos de regulación afectivos (Beebe \& Lachmann, 2014), fomentando el reconocimiento mutuo (Benjamin, 1988, 1995), tiene el propósito de involucrarse en toda esta complejidad y al mismo tiempo reconocer humildemente los límites inevitables (Cooper) que poco a poco se convierten en el desafío de la hazaña terapéutica. Trabajar de este modo requiere rigor y una implicación profunda por parte del terapeuta (Ehrenberg 1992, Ipp, 2010), que es lo que lo hace tan excitante; en cambio, uno debe estar dispuesto a hacerlo sin el paracaídas del diagnóstico, el soporte de las técnicas, los parámetros estándar y los objetivos especialmente prestablecidos (Ringstrom, 2012). El encuadre es crucial si uno tiene en mente que dentro del proceso surge un encuadre que respira sin rigidez y que la negociación del encuadre promueve los aspectos clave del proceso terapéutico (Bass, 2007). Dicho trabajo es "hecho a medida", un arte, sabiduría práctica, y debería también añadir que el proceso facilitado por este tipo de enfoque es único. Con un gran insight, en 1970 Bion ya habló de la opacidad de la memoria y el deseo: "...la capacidad de olvidar, la habilidad para evitar el deseo y el entendimiento, deben ser vistas como la disciplina esencial para el psicoanalista." (Bion, 1970, p. 51). Los pacientes deben considerar la posibilidad de que la activación potencial de los antiguos anhelos ocurra en el contexto de una relación realmente segura. Estoy de acuerdo con Mal en que los pacientes deberían experimentar al analista como genuinamente aliado con su interés pero -a pesar de nuestras mejores intenciones- esto solo es posible de una forma intermitente e imprecisa.

\section{REFERENCIAS}


Bass, A. (2007). When the frame doesn't fit the picture. Psychoanalytic Dialogues, 17, 1-27.

Beebe, B., Jaffe, J., Chen, H., Buck, K., Cohen, P., Feldstein, S., Andrews, H. (2008). Sixweek postpartum depressive symptoms and 4 -month mother-infant self- and interactive contingency. Infant Mental Health Journal, 29 (5), 442-471.

Beebe, B., Lachmann, F. (2014). The Origins of Attachment. Infant Research and Adult Treatment. New York, NY: Routledge.

Benjamin, J. (1988). The Bonds of Love. New York, NY: Pantheon.

Benjamin, J. (1995). Like Subjects, Love Objects: Essays on Recognition and Sexual Difference. New Haven, CT: Yale University Press.

Bion, W.R. (1970). Attention and Interpretation. London, Karnac Books.

Bromberg, P.M. (1998). Standing in the spaces: Essays on clinical process, trauma, and dissociation. Hillsdale, NJ: The Analytic Press.

Cooper, S. (2010). A disturbance in the field. New York, NY: Routledge.

Ehrenberg, D.B. (1992). The Intimate Edge: Extending the Reach of Psychoanalytic Interaction. New York: Norton.

Fogel, A. (1992). Co-regulation, perception and action. Human Movement Science, 11, 505523.

Ipp, H. (2010). Nell: A bridge to the amputated self: The impact of immigration on continuities and dicintinuities of self. International Journal of Psychoanalytic Self Psychology, 5, 373-386.

Mitchell, S.A. (1997), Influences and Autonomy in Psychoanalysis. Hillsdale, NJ: The Analytic Press.

Ringstrom, P. (2012). Principles of imprvisation: A model of therapeutic play in relarional psychoanalysis. In L .Aron \& A. Harris (eds), Relational psychoanalysis: Evolution of process (Vol. 5, pp. 447-478). New York, NY: Routledge.

Slavin, M. (2007). "Broadening the Context of Human Intersubjectivity: Making Meaning and Navigating Relational Conflict in Evolutionary Biological and Clinical Perspective". Presented at the IAPSP International Conference, Los Angeles, USA, October 2007.

Stern, D.N. (2004). The Present Moment in Psychotherapy and Everyday Life. New York, NY: Norton.

Tornatore, G. (1998). The Legend of 1990. British Board Film Classification, 2015. 
Trevarthen, C. (1998). The concept and foundation of infant intersubjectivity. In S. Braten (Ed.), Intersubjective communication in early ontogeny. Cambridge, MA: Cambridge University Press.

Tronick, E. (2008). Regolazione emotiva nello sviluppo e nel processo terapeutico. Milano, Raffaello Cortina Editore.

Original recibido con fecha: 24/10/2015 Revisado: 19/02/2016 Aceptado: 28/02/2016 